\title{
Consensus disturbance rejection for Lipschitz nonlinear multi-agent systems with input delay: a DOBC approach
}

DOI:

10.1016/j.jranklin.2016.09.019

\section{Document Version}

Submitted manuscript

Link to publication record in Manchester Research Explorer

\section{Citation for published version (APA):}

Wang, C., Zuo, Z., Sun, J., Yang, J., \& Ding, Z. (2017). Consensus disturbance rejection for Lipschitz nonlinear multi-agent systems with input delay: a DOBC approach. Journal of the Franklin Institute, 354(1), 298-315. https://doi.org/10.1016/j.jranklin.2016.09.019

\section{Published in:}

Journal of the Franklin Institute

\section{Citing this paper}

Please note that where the full-text provided on Manchester Research Explorer is the Author Accepted Manuscript or Proof version this may differ from the final Published version. If citing, it is advised that you check and use the publisher's definitive version.

\section{General rights}

Copyright and moral rights for the publications made accessible in the Research Explorer are retained by the authors and/or other copyright owners and it is a condition of accessing publications that users recognise and abide by the legal requirements associated with these rights.

\section{Takedown policy}

If you believe that this document breaches copyright please refer to the University of Manchester's Takedown Procedures [http://man.ac.uk/04Y6Bo] or contact uml.scholarlycommunications@manchester.ac.uk providing relevant details, so we can investigate your claim.

\section{OPEN ACCESS}




\title{
Consensus disturbance rejection for Lipschitz nonlinear

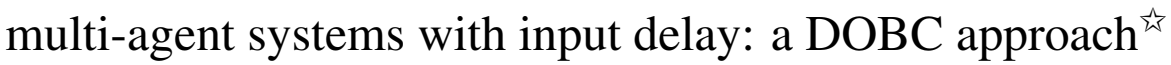

\author{
Chunyan Wang ${ }^{\mathrm{a}}$, Zongyu Zuo ${ }^{\mathrm{b}}$, Junyong Sun ${ }^{\mathrm{c}}$, Jun Yang ${ }^{\mathrm{d}}$, Zhengtao Ding ${ }^{\mathrm{a}, *}$ \\ ${ }^{a}$ School of Electrical and Electronic Engineering, University of Manchester, Sackville Street Building, \\ Manchester M13 9PL, UK \\ ${ }^{b}$ The Seventh Research Division, and Science and Technology on Aircraft Control Laboratory, Beihang \\ University, Beijing 100191, China \\ ${ }^{c}$ State Key Laboratory for Turbulence and Complex Systems, Department of Mechanics and Engineering \\ Science, College of Engineering, Peking University, Beijing 100871, P. R. China \\ ${ }^{d}$ The School of Automation, Southeast University, Key Laboratory of Measurement and Control of CSE, \\ Ministry of Education, Nanjing 210096, P. R. China
}

\begin{abstract}
In this paper, a new predictor-based consensus disturbance rejection method is proposed for high-order multi-agent systems with Lipschitz nonlinearity and input delay. First, a distributed disturbance observer for consensus control is developed for each agent to estimate the disturbance under the delay constraint. Based on the conventional predictor feedback approach, a non-ideal predictor based control scheme is constructed for each agent by utilizing the estimate of the disturbance and the prediction of the relative state information. Then, rigorous analysis is carried out to ensure that the extra terms associated with disturbances and nonlinear functions are properly considered. Sufficient conditions for the consensus of the multi-agent systems with disturbance rejection are derived based on the analysis in the framework of Lyapunov-Krasovskii functionals. A simulation example is included to demonstrate the performance of the proposed control scheme.
\end{abstract}

Keywords: Consensus, multi-agent systems, DOBC, Lipschitz nonlinearity, input delay

\footnotetext{
The material in this paper was not presented at any conference.

* Corresponding author

Email addresses: chunyan.wang-2@manchester.ac.uk (Chunyan Wang), zzybobby@buaa. edu. cn (Zongyu Zuo), s jymath@pku. edu. cn (Junyong Sun), j.yang84@seu.edu.cn (Jun Yang), zhengtao.ding@manchester.ac.uk (Zhengtao Ding)
} 


\section{Introduction}

In recent years, cooperative control has received considerable attention in control community. An important problem arising from cooperative control is to coordinate individual dynamic systems, which have the same or similar dynamics, to perform a common control task, and this topic is commonly known as consensus control [1]. The early results concentrated on consensus problems of simple agent dynamics such as single and double integrators [2, 3, 4], and then the results were extended to high-order linear systems [5, 6, 7, 8, 9] and also nonlinear systems [10, 11, 12]. Some applications in formation control [13, 14, 15, 16], synchronization [17, 18, 19, 20], and mechanical systems [21, 22], have also been investigated.

Since practical multi-agent systems often suffer from various external disturbances, considerable effort has been placed on consensus disturbance rejection problem. Some robust control methods, such as $H_{\infty}$ consensus control [23, 24], have been proved to be effective for disturbance rejection of the multi-agent systems with external disturbances bounded by $\mathrm{H}_{2}$ norms. However, disturbances in real engineering problems are often periodic and have inherent characteristics such as harmonics and unknown constant load [25]. For those kinds of disturbances, it is desirable by utilizing the disturbance information in the design of control input to cancel the disturbances directly. One common design method is to estimate the disturbance by using the measurements of states or outputs and then use the disturbance estimate to compensate the influence of the disturbance on the system, which is referred to as disturbance observer-based control (DOBC) [26]. Using DOBC, consensus of second-order multi-agent dynamical systems with exogenous disturbances was studied in [27, 28] for matched disturbances and in [29] for unmatched disturbances. Disturbance observer based tracking controllers for high-order integrator-type and general multi-agent systems were proposed in [30, 31], respectively. A systematic study on consensus disturbance rejection via disturbance observers could be found in [32]. Note that most existing results are limited to linear systems.

In reality, other than the external disturbances, time delays are inevitable in various practical engineering systems including networked control systems [33]. Some recent 
results for consensus control of multi-agent systems with delays can be found in [34, 35, 36, 37 and the references therein. It is generally recognized that the system with input delay is more involved than the system with state delay in control theory. A wide variety of predictor-based feedback approaches are effective for systems with input delay (see [38] and the references therein). However, there is a lack of study on consensus disturbance rejection for multi-agent systems with input delay which is more relevant from a practical point of view. The problem of consensus controller design with input delay is more involved due to the undesirable disturbances. For example, the model reduction method used in [36] cannot be applied for the consensus disturbance rejection design in the presence of disturbances.

Inspired by the previous discussions, in this paper, we consider the consensus disturbance rejection problem for Lipschitz nonlinear multi-agent systems with input delay based on the DOBC approach. Compared with the previous works, the key features of this paper are as follows: (1) Input delay is taken into account for the consensus disturbance rejection problem. Different from the conventional predictor feedback approach, a non-ideal predictor based control scheme is constructed for each agent by using the estimate of the disturbance and the prediction of the relative state. (2) Unlike [31], [32], where the agent dynamics are restricted to be linear, we consider the Lipschitz nonlinearity in the system dynamics. Rigorous analysis within the framework of Lyapunov-Krasovskii functionals is carried out to guarantee that the extra integral terms of the system state associated with nonlinear functions are properly considered.

The remainder of this paper is organized as follows. In Section 2, the problem formulation is introduced. Section 3 presents some notations and preliminary results. Section 4 presents the main results on the consensus disturbance rejection design. Simulation results are given in Section 5 Section 6 concludes the paper.

\section{Problem Statement}

In this paper, we consider the leader-follower consensus control of a group of $N$ agents. Assume that the dynamics of followers, labelled as $2,3, \ldots, N$, are described 
by

$$
\dot{x}_{i}(t)=A x_{i}(t)+\phi\left(x_{i}(t)\right)+B u_{i}(t-h)+D \omega_{i}(t),
$$

and the leader agent is indexed by 1 , whose dynamics are represented by

$$
\dot{x}_{1}(t)=A x_{1}(t)+\phi\left(x_{1}(t)\right)
$$

where $x_{i} \in \mathbb{R}^{n}$ denotes the state, $u_{i} \in \mathbb{R}^{m}$ denotes the control input, $x_{1} \in \mathbb{R}^{n}$ is the leader's state, $A \in \mathbb{R}^{n \times n}$ and $B \in \mathbb{R}^{n \times m}$ are constant matrices with $(A, B)$ being controllable, $h \in \mathbb{R}_{+}$is the constant and known input delay, $\omega_{i} \in \mathbb{R}^{s}$ is a disturbance that is generated by a linear exogenous system

$$
\dot{\omega}_{i}(t)=S \omega_{i}(t)
$$

65 $\mathbb{R}^{n}, \phi(0)=0$, is assumed to satisfy the Lipschitz condition as

$$
\|\phi(\alpha)-\phi(\beta)\| \leq \gamma\|\alpha-\beta\|, \forall \alpha, \beta \in \mathbb{R}^{n}
$$

where $\gamma>0$ is the Lipschitz constant.

Let $\xi_{i}=x_{i}-x_{1}, i=2,3, \cdots, N$ as the tracking errors. Then, based on the system dynamics (1) and (2), the error dynamics of the $i$ th agent can be obtained as

$$
\dot{\xi}_{i}(t)=A \xi_{i}(t)+\psi_{i}+B u_{i}(t-h)+D \omega_{i}(t)
$$

70 where $\psi_{i}=\phi\left(x_{i}(t)\right)-\phi\left(x_{1}(t)\right)$.

With the agent 1 as the leader, the control objective is to design a control input for each agent to follow the state of the leader $x_{1}$ under the disturbances. That is, under these control inputs, the following hold for any initial conditions,

$$
\lim _{t \rightarrow \infty}\left(x_{i}(t)-x_{1}(t)\right)=\lim _{t \rightarrow \infty} \xi_{i}(t)=0, \forall i=2,3, \cdots, N .
$$

We make two assumptions on the dynamics of the agents and the connections 75 among the agents.

Assumption 1. The disturbance is matched. i.e., there exist a matrix $F \in \mathbb{R}^{m \times s}$ such that $D=B F$. 
Assumption 2. The communication topology $\mathcal{G}$ contains a directed spanning tree with the leader as the root.

Remark 1. In this paper, the input delay under consideration is fixed and identical, and it is adopted for the convenience of presentation of the proposed design. The analysis presented in this paper could be extended to the case of time-varying delay, as long as the upper bound of the time delay is known.

Remark 2. The matching condition in Assumption 1 guarantees that the disturbance 85 act via the same channel as that of the control input. This assumption could be relaxed in some circumstances because unmatched disturbances under uncertain conditions may be converted to the matched ones based on output regulation theory [32]. Furthermore, the disturbance condition given in $(3)$ is commonly used for disturbance rejection and output regulation. Many kinds of disturbances in engineering can be described by this model. For instance, unknown constant disturbances or harmonics with unknown amplitudes and phases, belong to the allowed class of disturbances.

\section{Preliminary Results}

\subsection{Notations}

Throughout the paper, let $\mathbb{R}^{n \times m}$ and $\mathbb{R}^{n}$ represent a set of $n \times m$ real matrices and $n$-dimensional column vectors, and $0_{n \times m}$ denotes the $n \times m$ matrices with all zeros. Let 1 and $I$ represent a column vector with all entries equal to one and the identity matrix with appropriate dimension, respectively. $\mathcal{L}_{2}^{p}[0, \infty)$ denotes the space of $p$ dimensional square integrable functions over $[0, \infty)$. Given a real matrix $X \in \mathbb{R}^{n \times m}$ and vector $x \in \mathbb{R}^{n},\|X\|_{F}$ denotes the Frobenius norm, and $\|x\|$ is the Euclidean norm. The symbol $\otimes$ denotes the Kronecker product of matrices, and the notation $\operatorname{diag}\left(\pi_{i}\right)$ denotes a block-diagonal matrix with $\pi_{i}, i=1,2, \cdots, N$, on the diagonal. The matrix inequality $A>B$ means that $A-B$ is positive definite. 


\subsection{Graph theory}

The communication topology among the agents is described by the directed graph $\mathcal{G} \triangleq(\mathcal{V}, \mathcal{E})$, in which $\mathcal{V} \triangleq\{1, \cdots, N\}$ is the set of nodes, and $\mathcal{E} \subseteq \mathcal{V} \times \mathcal{V}$ is the set of edges with the ordered pair of nodes. A vertex represents an agent, and each edge represents a connection. Associated with the communication graph is its adjacency matrix $\mathcal{A}=\left[a_{i j}\right] \in \mathbb{R}^{N \times N}$, where the element $a_{i j}$ denotes the connection between the agent $i$ and agent $j . a_{i j}=1$ if $(j, i) \in \mathcal{E}$, otherwise is zero, and $a_{i i}=0$ for all nodes with the assumption that there exists no self loop. In the directed graph $\mathcal{G}$, $(i, j) \in \mathcal{E}$ denotes that the $j$ th agent can obtain the information from the $i$ th agent, but not vice versa. A directed path on the graph $\mathcal{G}$ from node $i_{1}$ to node $i_{s}$ is a sequence of ordered edges as $\left(i_{1}, i_{2}\right),\left(i_{2}, i_{3}\right), \cdots,\left(i_{s-1}, i_{s}\right)$. A directed graph that contains a spanning tree is that there exists a node called the root, and this root has a directed path to every other node of the graph. The Laplacian matrix $\mathcal{L}=\left[l_{i j}\right] \in \mathbb{R}^{N \times N}$ is defined by $l_{i i}=\sum_{j=1}^{N} a_{i j}$ and $l_{i j}=-a_{i j}$ when $i \neq j$. For a directed graph, the Laplacian matrix $\mathcal{L}$ has the following properties.

Lemma 1 ([3, 39]). The Laplacian matrix $\mathcal{L}$ of a directed graph $\mathcal{G}$ has at least one zero eigenvalue with a corresponding right eigenvector $\mathbf{1}=[1,1, \ldots, 1]^{T}$ and all nonzero eigenvalues have positive real parts. Furthermore, zero is a simple eigenvalue of $\mathcal{L}$ if and only if $\mathcal{G}$ has a directed spanning tree.

Because the leader has no neighbours, the Laplacian matrix $\mathcal{L}$ of $\mathcal{G}$ has the following structure

$$
\mathcal{L}=\left[\begin{array}{cc}
0 & 0_{1 \times(N-1)} \\
\mathcal{L}_{2} & \mathcal{L}_{1}
\end{array}\right]
$$

where $\mathcal{L}_{1} \in \mathbb{R}^{(N-1) \times(N-1)}$ and $\mathcal{L}_{2} \in \mathbb{R}^{(N-1) \times 1}$. From Lemma 1 , it is obvious that $\mathcal{L}_{1}$ is a nonsingular M-matrix. We also have the following result for $\mathcal{L}_{1}$ :

Lemma 2 ([40]). For the nonsingular M-matrix $\mathcal{L}_{1}$, there exists a positive diagonal matrix $G$ such that

$$
G \mathcal{L}_{1}+\mathcal{L}_{1}^{T} G \geq r_{0} I
$$


for some positive constant $r_{0}$. It is also shown that $G$ can be constructed by let-

125 ting $G=\operatorname{diag}\left\{q_{2}, q_{3}, \cdots, q_{N}\right\}=(\operatorname{diag}(\pi))^{-1}$, where $\pi=\left[\pi_{2}, \pi_{3}, \cdots, \pi_{N}\right]^{T}=$ $\left(\mathcal{L}_{1}^{T}\right)^{-1}[1,1, \cdots, 1]^{T}$.

\subsection{Predictor-based feedback approach}

In this subsection, we recall the well-known predictor-based feedback approach [41, 42]. Consider a linear input-delayed system

$$
\dot{x}(t)=A x(t)+B u(t-h),
$$

where $x \in \mathbb{R}^{n}$ denotes the state, $u \in \mathbb{R}^{m}$ denotes the control input, $A \in \mathbb{R}^{n \times n}$ and $B \in \mathbb{R}^{n \times m}$ are constant matrices, $h \in \mathbb{R}_{+}$is input delay, which is known and constant. We design the feedback controller as

$$
u(t)=K x(t+h)
$$

Then the finite-dimensional closed-loop system can be expressed as

$$
\dot{x}(t)=(A+B K) x(t) .
$$

If we could find a possible control gain matrix $K$ to stabilize the closed-loop system, then the control design problem is solved. However, it is unrealistic since the state information $x$ at time $t+h$ cannot be obtained with direct measurement. A feasible way is to calculate the vector $x(t+h)$ as follows [41]:

$$
\begin{aligned}
x(t+h) & =e^{A(t+h)} x(0)+\int_{0}^{t+h} \mathrm{e}^{A(t+h-\tau)} B u(\tau-h) \mathrm{d} \tau \\
& =e^{A h} x(t)+\int_{t-h}^{t} \mathrm{e}^{A(t-\tau)} B u(\tau) \mathrm{d} \tau .
\end{aligned}
$$

Therefore we can express the controller with an ideal predictor

$$
u(t)=K\left(e^{A h} x(t)+\int_{t-h}^{t} \mathrm{e}^{A(t-\tau)} B u(\tau) \mathrm{d} \tau\right) .
$$

Based on the problem conditions, the multi-agent systems considered in this work are nonlinear and subject to deterministic disturbances with unknown bounds.

Before moving into the observer and controller design, a couple of preliminary results are presented below for the consensus analysis. 
Lemma 3 (Jensen's Inequality, [43]). For a positive definite matrix $P$, and a function $x:[a, b] \rightarrow \mathbb{R}^{n}$, with $a, b \in \mathbb{R}$ and $b>a$, the following inequality holds:

$$
\left(\int_{a}^{b} x^{T}(\tau) \mathrm{d} \tau\right) P\left(\int_{a}^{b} x(\tau) \mathrm{d} \tau\right) \leq(b-a) \int_{a}^{b} x^{T}(\tau) P x(\tau) \mathrm{d} \tau .
$$

Lemma 4 ([44, 45]). For a positive definite matrix $P$, the following identity holds

$$
e^{A^{T} t} P e^{A t}-e^{\alpha t} P=-e^{\alpha t} \int_{0}^{t} e^{-\alpha \tau} e^{A^{T} \tau} R e^{A \tau} \mathrm{d} \tau
$$

where

$$
R=-A^{T} P-P A+\alpha P .
$$

Furthermore, if $R$ is positive definite, $\forall t>0$,

$$
e^{A^{T} t} P e^{A t}<e^{\alpha t} P
$$

Lemma 5. For any given $a, b \in \mathbb{R}^{n}$, we have

$$
2 a^{T} S Q b \leq a^{T} S P S^{T} a+b^{T} Q^{T} P^{-1} Q b,
$$

where $P>0, S$ and $Q$ have appropriate dimensions.

\section{Main Results}

The disturbance rejection design consists of disturbance estimation and rejection. The estimation is based on the relative state information obtained through the communication network. It is assumed that the $i$ th agent collects the relative state information of its neighbouring agents as

$$
\zeta_{i}(t)=\sum_{j=1}^{N} a_{i j}\left(x_{i}(t)-x_{j}(t)\right), \forall i=2,3, \cdots, N .
$$

From the relationship between $\mathcal{A}$ and $\mathcal{L}$, it is easy to see that $\zeta_{i}(t)=\sum_{j=2}^{N} l_{i j} \xi_{j}(t)$.

${ }_{135}$ The disturbance estimation and rejection proposed in this paper will be designed based on relative state information $\zeta_{i}(t)$. 


\subsection{Controller and observer design}

The control input for disturbance rejection is designed as follows:

$$
u_{i}(t)=c K \chi_{i}(t)-F e^{S h} \hat{\omega}_{i}(t)
$$

where $\chi_{i}(t)$ and $\hat{\omega}_{i}(t)$ are generated by

$$
\begin{aligned}
\chi_{i}(t)= & e^{A h} \zeta_{i}(t)+\sum_{j=2}^{N} l_{i j} \int_{t-h}^{t} e^{A(t-\tau)} c B K \chi_{j}(t) \mathrm{d} \tau, \\
\hat{\omega}_{i}(t)= & \eta_{i}(t)+L \zeta_{i}(t), \\
\dot{\eta}_{i}(t)= & S\left(\eta_{i}(t)+L \zeta_{i}(t)\right)-L B F \sum_{j=2}^{N} l_{i j}\left(\eta_{j}(t)+L \zeta_{j}(t)\right) \\
& -L A \zeta_{i}(t)-L B \sum_{j=2}^{N} l_{i j} u_{j}(t-h),
\end{aligned}
$$

where $c \geq 2 q_{\max } / r_{0}$ is a positive real constant with $q_{\max }=\max \left\{q_{2}, q_{3}, \cdots, q_{N}\right\}, K$ and $L$ are constant gain matrices to be designed later.

Remark 3. The integral term of $\chi_{i}(t)$ is added in the controller design to offset the adverse effect of the time delay. If the nonlinear and disturbance terms in (1) are absent, $\chi_{i}(t)$ is an ideal predictor of the relative state information of the $i$ th agent. Due to the presence of disturbance, it is a non-ideal prediction of the relative state information. Furthermore, (12)-(13) are referred to as a distributed predictor-based consensus disturbance observer, which is only dependent on the relative state information, and independent of the information of the local state.

Let $z_{i}(t)=\omega_{i}(t)-\hat{\omega}_{i}(t)$. A direct evaluation gives that

$$
\begin{aligned}
\dot{z}_{i}(t) & =S \omega_{i}(t)-\dot{\eta}_{i}(t)-L \sum_{j=2}^{N} l_{i j} \dot{\xi}_{j}(t) \\
& =S z_{i}(t)-L \sum_{j=2}^{N} l_{i j} \psi_{j}-L B F \sum_{j=2}^{N} l_{i j} z_{j}(t),
\end{aligned}
$$

which can be written in the compact form as

$$
\dot{z}(t)=\left(I_{N-1} \otimes S\right) z(t)-\left(\mathcal{L}_{1} \otimes L B F\right) z(t)-\left(\mathcal{L}_{1} \otimes L\right) \Psi,
$$


where $\Psi=\left[\psi_{2}^{T}, \psi_{3}^{T}, \cdots, \psi_{N}^{T}\right]^{T}$.

With the control input (10), the closed-loop dynamics of each agent in (4) can be written as

$$
\begin{aligned}
\dot{\xi}_{i}(t)= & A \xi_{i}(t)+\psi_{i}+B F e^{S h} z_{i}(t-h)+c B K e^{A h} \sum_{j=2}^{N} l_{i j} \xi_{j}(t-h) \\
& +c B K \sum_{j=2}^{N} l_{i j} \int_{t-h}^{t} e^{A(t-\tau)} c B K \chi_{j}(\tau-h) \mathrm{d} \tau,
\end{aligned}
$$

where we have used $\omega_{i}(t)=e^{S h} \omega_{i}(t-h)$ and $D=B F$.

From the error dynamics (4), we have

$$
\xi_{i}(t)=e^{A h} \xi_{i}(t-h)+\int_{t-h}^{t} e^{A(t-\tau)}\left(\psi_{i}+B u_{i}(\tau-h)+D \omega_{i}(\tau)\right) \mathrm{d} \tau .
$$

Invoking (17) into (16), we obtain

$$
\begin{aligned}
\dot{\xi}_{i}(t)= & A \xi_{i}(t)+c B K \sum_{j=2}^{N} l_{i j} \xi_{j}(t)+\psi_{i}+B F e^{S h} z_{i}(t-h) \\
& -c B K \sum_{j=2}^{N} l_{i j} \int_{t-h}^{t} e^{A(t-\tau)}\left(\psi_{j}+B F e^{S h} z_{j}(\tau-h)\right) \mathrm{d} \tau .
\end{aligned}
$$

Let $\xi=\left[\xi_{2}^{T}, \xi_{3}^{T}, \cdots, \xi_{N}^{T}\right]^{T}, z=\left[z_{2}^{T}, z_{3}^{T}, \cdots, z_{N}^{T}\right]^{T}$. The error dynamics of $\xi(t)$ can be written in the compact form as

$$
\dot{\xi}(t)=\left(I \otimes A+c \mathcal{L}_{1} \otimes B K\right) \xi(t)+\Psi+\left(I \otimes B F e^{S h}\right) z(t-h)+\Delta_{1}+\Delta_{2},
$$

where

$$
\begin{aligned}
& \Delta_{1}=-\left(c \mathcal{L}_{1} \otimes B K\right) \int_{t-h}^{t}\left(I \otimes e^{A(t-\tau)}\right) \Psi \mathrm{d} \tau \\
& \Delta_{2}=-\left(c \mathcal{L}_{1} \otimes B K\right) \int_{t-h}^{t}\left(I \otimes e^{A(t-\tau)} B F e^{S h}\right) z(\tau-h) \mathrm{d} \tau .
\end{aligned}
$$

For the convenience, let $\Delta_{1}=\left[\delta_{2}^{T}, \delta_{3}^{T}, \cdots, \delta_{N}^{T}\right]^{T}$ and $\Delta_{2}=\left[\bar{\delta}_{2}^{T}, \bar{\delta}_{3}^{T}, \cdots, \bar{\delta}_{N}^{T}\right]^{T}$.

Next, we will design the control gain $K$ and the observer gain $L$. With the control law shown in $(10), K$ and $L$ are chosen as

$$
\begin{aligned}
& K=-\gamma_{1} B^{T} P, \\
& L=\gamma_{2} c Q^{-1} D^{T},
\end{aligned}
$$


where $\gamma_{1}, \gamma_{2}$ are positive parameters to be chosen, and $P>0, Q>0$ are constant matrices to be designed.

In order to obtain the main results, the bounds on $\left\|\Delta_{1}\right\|^{2}$ and $\left\|\Delta_{2}\right\|^{2}$ are given in the following lemma.

Lemma 6. For the terms $\Delta_{1}$ and $\Delta_{2}$ in the error dynamics $(19)$, bounds can be established as

$$
\begin{aligned}
& \left\|\Delta_{1}\right\|^{2} \leq \rho_{1} \int_{t-h}^{t} \xi^{T}(\tau) \xi(\tau) \mathrm{d} \tau \\
& \left\|\Delta_{2}\right\|^{2} \leq \rho_{2} \int_{t-h}^{t} z^{T}(\tau-h) z(\tau-h) \mathrm{d} \tau
\end{aligned}
$$

where

$$
\begin{aligned}
& \rho_{1}=(N-1) c^{2} \gamma_{1}^{2} h \rho^{2} e^{\alpha_{2} h} \gamma^{2}\left\|\mathcal{L}_{1}\right\|_{F}^{2}, \\
& \rho_{2}=(N-1) h \alpha_{1} c^{2} \gamma_{1}^{2} \rho^{2} e^{\left(\alpha_{0}+\alpha_{2}\right) h}\left\|\mathcal{L}_{1}\right\|_{F}^{2},
\end{aligned}
$$

with $\rho, \alpha_{0}, \alpha_{1}, \alpha_{2}$ being positive numbers such that

$$
\begin{aligned}
\rho^{2} I & \geq P B B^{T} B B^{T} P, \\
\alpha_{0} & >\lambda_{\max }\left(S+S^{T}\right), \\
\alpha_{1} & \geq \lambda_{\max }\left(F^{T} B^{T} B F\right), \\
\alpha_{2} & >\lambda_{\max }\left(A+A^{T}\right) .
\end{aligned}
$$

Proof. See the Appendix.

\subsection{Consensus analysis}

The following theorem presents sufficient conditions to ensure that the consensus disturbance rejection problem is solved by using the control algorithm $(10)$ with the control gain $K$ and the observer gain $L$ in 20, 21 .

Theorem 7. For multi-agent systems (1)-(2) with Assumptions 1 and 2 the consensus disturbance rejection problem can be solved by the control algorithm (10) with (20)(21) if there exists positive definite matrices $P, Q$ and constants $\rho, \gamma_{1}, \gamma_{2}>0$, such 
that

$$
\begin{aligned}
& \rho W-B B^{T} \geq 0, \\
& {\left[\begin{array}{cc}
A W+W A^{T}-2 \gamma_{1} B B^{T}+H & W \\
W & -\epsilon_{1}^{-1}
\end{array}\right]<0,} \\
& Q S+S^{T} Q-2 \gamma_{2} D^{T} D+\epsilon_{2} I<0,
\end{aligned}
$$

are satisfied with $W=P^{-1}$ and

$$
\begin{aligned}
& H=\left(\kappa_{1}+\kappa_{2}+\kappa_{3}+\kappa_{4}\right) I \\
& \epsilon_{1}=\left(\kappa_{1}^{-1}+c \gamma_{2} \kappa_{5}^{-1} \sigma_{\max }^{2}\left(\mathcal{L}_{1}\right)\right) \gamma^{2}+\rho_{1} \pi_{\min }^{-1} \pi_{\max } \kappa_{3}^{-1} e^{h}, \\
& \epsilon_{2}=\pi_{\max } \pi_{\min }^{-1}\left(\alpha_{1} \kappa_{2}^{-1} e^{\left(\alpha_{0}+1\right) h}+c \gamma_{2} \kappa_{5} \lambda_{\max }\left(D^{T} D\right)+e^{h} \kappa_{4}^{-1} \rho_{2}\right),
\end{aligned}
$$

where $\pi_{\min }=\min \left\{\pi_{2}, \pi_{3}, \cdots, \pi_{N}\right\}, \pi_{\max }=\max \left\{\pi_{2}, \pi_{3}, \cdots, \pi_{N}\right\}$.

PROOF. To start the consensus analysis, we try a Lyapunov function candidate

$$
V_{0}=\xi^{T}(G \otimes P) \xi+z^{T}(G \otimes Q) z+\sigma_{0} e^{h} \int_{t-h}^{t} e^{\tau-t} z^{T}(\tau) z(\tau) \mathrm{d} \tau
$$

where $\sigma_{0}$ is a positive value to be chosen later. 
The derivative of $V_{0}$ along the trajectory of $(15)$ and $(19)$ can be obtained as

$$
\begin{aligned}
\dot{V}_{0}= & \xi^{T}\left(G \otimes\left(P A+A^{T} P\right)-c \gamma_{1}\left(G \mathcal{L}_{1}+\mathcal{L}_{1}^{T} G\right) \otimes P B B^{T} P\right) \xi \\
& +2 \sum_{i=2}^{N} \frac{1}{\pi_{i}} \xi_{i}^{T} P\left(B F e^{S h} z_{i}(t-h)+\psi_{i}+\delta_{i}+\bar{\delta}_{i}\right) \\
& +z^{T}(t)\left(G \otimes\left(Q S+S^{T} Q\right)-c \gamma_{2}\left(G \mathcal{L}_{1}+\mathcal{L}_{1}^{T} G\right) \otimes D^{T} D\right) z(t) \\
& -2 \gamma_{2} c z^{T}(t)\left(G \mathcal{L}_{1} \otimes D^{T}\right) \Psi-\sigma_{0} e^{h} \int_{t-h}^{t} e^{\tau-t} z^{T}(\tau) z(\tau) \mathrm{d} \tau \\
& +\sigma_{0} e^{h} z^{T}(t) z(t)-\sigma_{0} z^{T}(t-h) z(t-h) \\
\leq & \xi^{T}\left(G \otimes\left(P A+A^{T} P+\left(\kappa_{1}+\kappa_{2}+\kappa_{3}+\kappa_{4}\right) P P\right)-\gamma_{1} c r_{0} I \otimes P B B^{T} P\right) \xi \\
& +\frac{\gamma^{2}}{\kappa_{1}} \sum_{i=2}^{N} \frac{1}{\pi_{i}} \xi_{i}^{T} \xi_{i}+\frac{\alpha_{1}}{\kappa_{2}} e^{\alpha_{0} h} \sum_{i=2}^{N} \frac{1}{\pi_{i}} z_{i}^{T}(t-h) z_{i}(t-h)+\frac{\left\|\Delta_{1}\right\|^{2}}{\kappa_{3} \pi_{\min }}+\frac{\left\|\Delta_{2}\right\|^{2}}{\kappa_{4} \pi_{\min }} \\
& +z^{T}(t)\left(G \otimes\left(Q S+S^{T} Q+\frac{\pi_{\max }}{\pi_{\min }} \gamma_{2} c \kappa_{5} \lambda_{\max }\left(D^{T} D\right) I\right)-\gamma_{2} c r_{0} I \otimes D^{T} D\right) z(t) \\
& -\sigma_{0} z^{T}(t-h) z(t-h)+\sigma_{0} e^{h} z^{T}(t) z(t)+\frac{\gamma_{2} c \gamma^{2}}{\kappa_{5}} \sigma_{\max }^{2}\left(\mathcal{L}_{1}\right) \sum_{i=2}^{N} \frac{1}{\pi_{i}} \xi_{i}^{T} \xi_{i} \\
\leq & \xi^{T}\left(G \otimes\left(P A+A^{T} P-2 \gamma_{1} P B B^{T} P+\left(\kappa_{1}+\kappa_{2}+\kappa_{3}+\kappa_{4}\right) P P+\sigma_{1} I\right)\right) \xi \\
& +z^{T}(t)\left(G \otimes\left(Q S+S^{T} Q-2 \gamma_{2} D^{T} D+\sigma_{11} I\right)\right) z(t)+\frac{\left\|\Delta_{1}\right\|^{2}}{\kappa_{3} \pi_{\min }}+\frac{\left\|\Delta_{2}\right\|^{2}}{\kappa_{4} \pi_{\min }} \\
& +\left(\alpha_{1} \kappa_{2}^{-1} \pi_{\min }^{-1} e^{\alpha_{0} h}-\sigma_{0}\right) z^{T}(t-h) z(t-h),
\end{aligned}
$$

where $\sigma_{1}=\left(\kappa_{1}^{-1}+\gamma_{2} c \kappa_{5}^{-1} \sigma_{\max }^{2}\left(\mathcal{L}_{1}\right)\right) \gamma^{2}, \sigma_{11}=\sigma_{0} \pi_{\max } e^{h}+\gamma_{2} c \kappa_{5} \pi_{\max } \pi_{\min }^{-1} \lambda_{\max }\left(D^{T} D\right)$. Lemmas 2, 4 and 5 are used in above derivation.

By choosing $\sigma_{0}=\alpha_{1} \kappa_{2}^{-1} \pi_{\min }^{-1} e^{\alpha_{0} h}$, the derivative of $V_{0}$ could be written as

$$
\begin{aligned}
\dot{V}_{0} \leq & \xi^{T}\left(G \otimes\left(P A+A^{T} P-2 \gamma_{1} P B B^{T} P+\left(\kappa_{1}+\kappa_{2}+\kappa_{3}+\kappa_{4}\right) P P+\sigma_{1} I\right)\right) \xi \\
& +z^{T}\left(G \otimes\left(Q S+S^{T} Q-2 \gamma_{2} D^{T} D+\sigma_{2} I\right)\right) z+\frac{\rho_{1}}{\kappa_{3} \pi_{\min }} \int_{t-h}^{t} \xi^{T}(\tau) \xi(\tau) \mathrm{d} \tau \\
& +\frac{\rho_{2}}{\kappa_{4} \pi_{\min }} \int_{t-h}^{t} z^{T}(\tau-h) z(\tau-h) \mathrm{d} \tau
\end{aligned}
$$

where $\sigma_{2}=\pi_{\max } \pi_{\min }^{-1}\left(\alpha_{1} \kappa_{2}^{-1} e^{\left(\alpha_{0}+1\right) h}+\gamma_{2} c \kappa_{5} \lambda_{\max }\left(D^{T} D\right)\right)$.

To deal with the first integral term shown in $(33)$, we consider the following Krasovskii 
functional

$$
V_{1}=e^{h} \int_{t-h}^{t} e^{\tau-t} \xi^{T}(\tau) \xi(\tau) \mathrm{d} \tau
$$

With the direct calculations as

$$
\begin{aligned}
\dot{V}_{1} & =-e^{h} \int_{t-h}^{t} e^{\tau-t} \xi^{T}(\tau) \xi(\tau) \mathrm{d} \tau+e^{h} \xi^{T}(t) \xi(t)-\xi^{T}(t-h) \xi(t-h) \\
& \leq-\int_{t-h}^{t} \xi^{T}(\tau) \xi(\tau) \mathrm{d} \tau+e^{h} \xi^{T}(t) \xi(t) .
\end{aligned}
$$

Similarly, the second integral term in 33 is coped with as

$$
V_{2}=e^{h} \int_{t-h}^{t} z^{T}(\tau) z(\tau) \mathrm{d} \tau+e^{h} \int_{t-h}^{t} e^{\tau-t} z^{T}(\tau-h) z(\tau-h) \mathrm{d} \tau .
$$

With the derivative as

$$
\begin{aligned}
\dot{V}_{2} & =-e^{h} \int_{t-h}^{t} e^{\tau-t} z^{T}(\tau-h) z(\tau-h) \mathrm{d} \tau+e^{h} z^{T}(t) z(t)-z^{T}(t-2 h) z(t-2 h) \\
& \leq-\int_{t-h}^{t} z^{T}(\tau-h) z(\tau-h) \mathrm{d} \tau+e^{h} z^{T}(t) z(t)
\end{aligned}
$$

Let

$$
V=V_{0}+\rho_{1} \pi_{\min }^{-1} \kappa_{3}^{-1} V_{1}+\rho_{2} \pi_{\min }^{-1} \kappa_{4}^{-1} V_{2}
$$

A direct evaluation gives that

$$
\dot{V} \leq \xi^{T}(t)\left(G \otimes P_{1}\right) \xi(t)+z^{T}(t)\left(G \otimes Q_{1}\right) z(t),
$$

where

$$
\begin{aligned}
& P_{1}=P A+A^{T} P-2 \gamma_{1} P B B^{T} P+\left(\kappa_{1}+\kappa_{2}+\kappa_{3}+\kappa_{4}\right) P P+\epsilon_{1} I, \\
& Q_{1}=Q S+S^{T} Q-2 \gamma_{2} D^{T} D+\epsilon_{2} I .
\end{aligned}
$$

The condition in 28) is equivalent to the condition specified in 24). With 38) and (39), it can be shown by Schur Complement that conditions (29) and 30) are respectively equivalent to $P_{1}<0$ and $Q_{1}<0$, which further implies from 37) that $\dot{V}(t)<0$. Thus, the error dynamics systems 4 are globally asymptotically stable at the origin, which implies that the consensus disturbance rejection of the multi-agent systems (1)-2) is achieved. This completes the proof. 


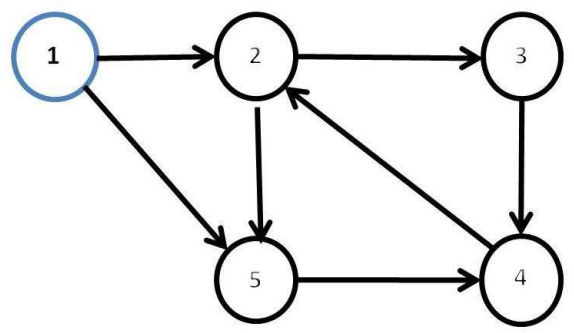

Figure 1: Communication topology.

170 Remark 4. It is observed that 29] and 30] are more likely to be satisfied if the values of $\rho, \gamma_{1}, \gamma_{2}, \kappa_{i}, i=1,2, \cdots, 5$ are small. Therefore, the step by step algorithm designed in [46] for finding a feasible solution of the conditions may also be applied here. Furthermore, since the values of $h$ and $\gamma$ are fixed and they are not the decision variables of the LMIs, a feasible solution may not exist if the values of $h$ and $\gamma$ are too 175 large.

\section{Simulation}

In this section, we will demonstrate the consensus disturbance rejection method under the leader-follower setup of five subsystems subject to the connection topology specified by the following adjacency matrix

$$
\mathcal{A}=\left[\begin{array}{lllll}
0 & 0 & 0 & 0 & 0 \\
1 & 0 & 0 & 1 & 0 \\
0 & 1 & 0 & 0 & 0 \\
0 & 0 & 1 & 0 & 1 \\
1 & 1 & 0 & 0 & 0
\end{array}\right] .
$$

Note that the first row all are zeros, as the agent indexed by 1 is taken as the leader. The communication graph is shown in Figure 1, from which it shows that only the followers indexed by 2 and 5 can get access to the leader and the communication topology contains a directed spanning tree. The dynamics of the $i$ th agent are described by (1), 
with

$$
A=\left[\begin{array}{cccc}
-0.2 & -2 & 2.4 & 1 \\
0 & -1.4 & 0.8 & 0.2 \\
0 & -1.3 & -0.8 & -2 \\
0.2 & 0 & 1 & -0.3
\end{array}\right], B=\left[\begin{array}{c}
0 \\
0 \\
0 \\
0.1
\end{array}\right], \phi\left(x_{i}\right)=g\left[\begin{array}{c}
\sin \left(x_{i 1}\right) \\
0 \\
0 \\
0
\end{array}\right] .
$$

In this scenario, it is supposed that external disturbance and time delay exist in the control channel. The external disturbance $w_{i}(t)$ is generated by 3 with

$$
S=\left[\begin{array}{cc}
0 & 0.1 \\
-0.1 & 0
\end{array}\right], F=\left[\begin{array}{ll}
1 & 0
\end{array}\right],
$$

which represents an external periodic disturbance with known frequency but without any information of its magnitude and phase. The input delay of each agent is $0.03 \mathrm{~s}$, and the Lipschitz constant is $\gamma=g=0.01$. It can be checked that both Assumptions $180 \quad 1$ and 2 are satisfied.

The Laplacian matrix $\mathcal{L}_{1}$ associated with $\mathcal{A}$ is that

$$
\mathcal{L}_{1}=\left[\begin{array}{cccc}
2 & 0 & -1 & 0 \\
-1 & 1 & 0 & 0 \\
0 & -1 & 2 & -1 \\
-1 & 0 & 0 & 2
\end{array}\right]
$$

Following Lemma 2 we obtain that $G=\operatorname{diag}\left\{\begin{array}{llll}0.3846 & 0.3571 & 0.5556 & 0.7143\end{array}\right\}$ and $r_{0}=0.2573$. With $p_{\max }=0.7143$ and $2 p_{\max } / r_{0}=5.5523$, we set $c=6$ in the control input 10.

The initial states of agents are chosen randomly within $[-5,5]$, and $u(\theta)=0, \forall \theta \in$ $[-h, 0]$. With $\rho=0.01, \gamma_{1}=10, \gamma_{2}=100$, feasible solutions of the feedback gain $K$ and the observer gain $L$ are found to be

$$
K=\left[\begin{array}{llll}
0.0059 & -0.024 & -0.0016 & -0.026
\end{array}\right], \quad L=\left[\begin{array}{cccc}
0 & 0 & 0 & 9.5836 \\
0 & 0 & 0 & 5.8656
\end{array}\right]
$$
ures 2-5 show the tracking errors between the four followers and the leader. The disturbance observation errors are shown in Figure 6. From the results shown in these 


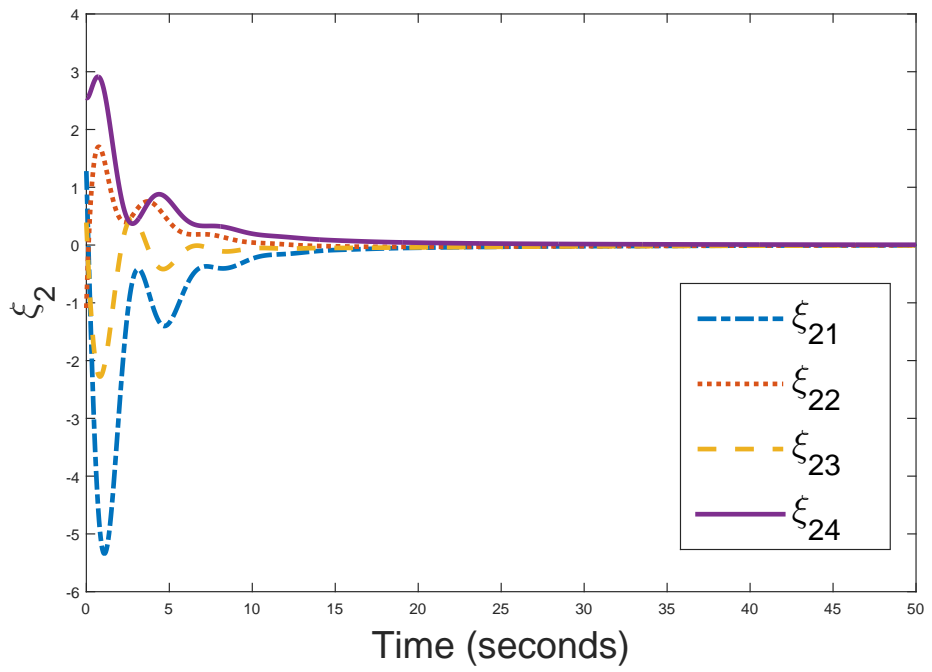

Figure 2: The evolutions of tracking errors $\xi_{2}$.

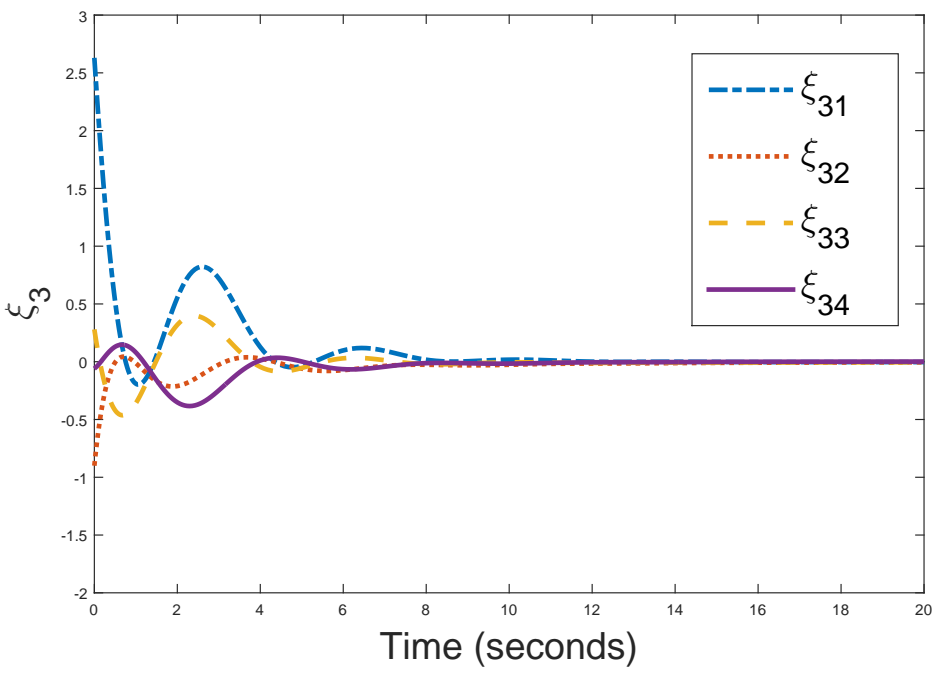

Figure 3: The evolutions of tracking errors $\xi_{3}$. 


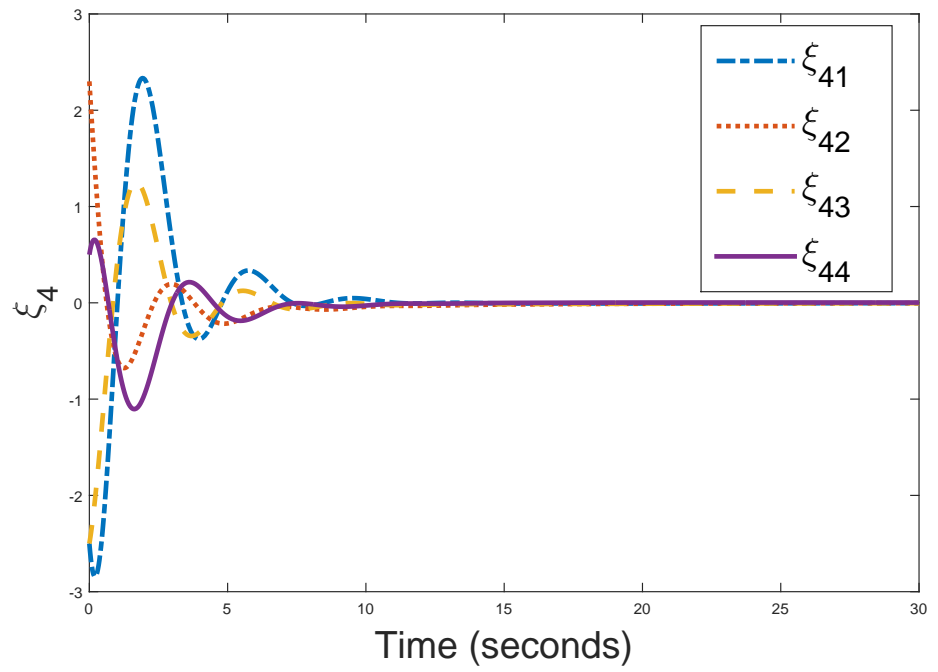

Figure 4: The evolutions of tracking errors $\xi_{4}$.

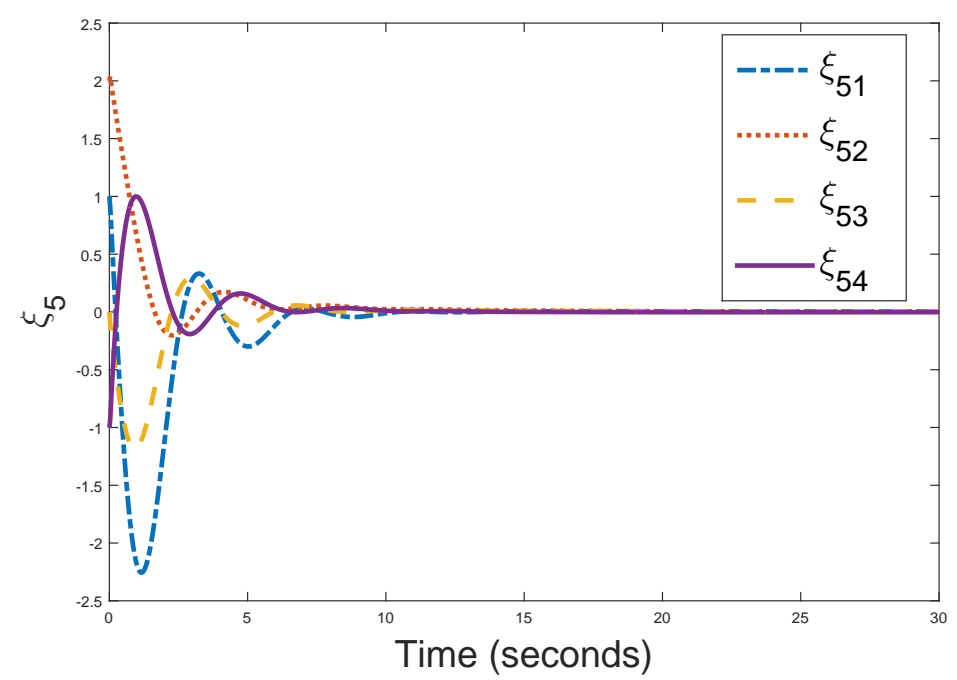

Figure 5: The evolutions of tracking errors $\xi_{5}$. 

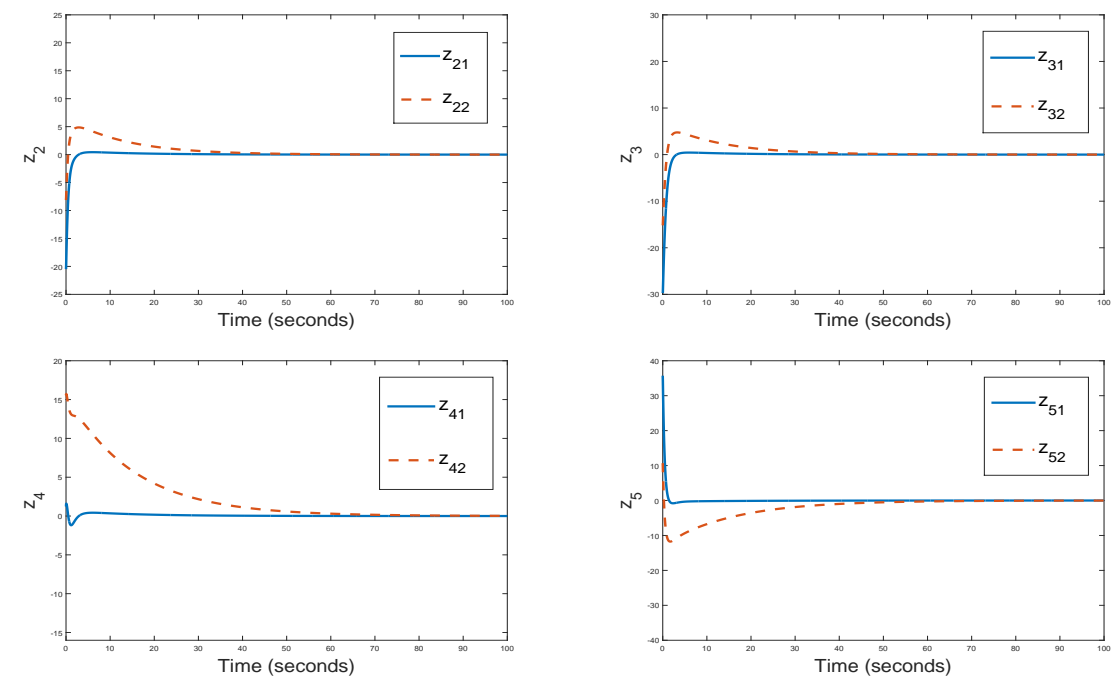

Figure 6: The estimation errors of the disturbance observers.

figures, it can be seen clearly that all the five agents reach consensus although they are under different disturbances. Therefore, the conditions specified in Theorem 7 are sufficient to guarantee the consensus disturbance rejection.

Moreover, as only the Lipschitz constant $\gamma$ is used for the disturbance observer design and the exact information of the nonlinear functions is not required, this leads to conservatism in the presented conditions. With the same control gain, the consensus disturbance rejection is still achieved for the multi-agent systems with a larger Lipschitz constant.

\section{Conclusion}

In this paper, we have addressed the consensus disturbance rejection problem for Lipschitz nonlinear multi-agent systems with input delay under the directed communication graph. The input delay may represent some delays in the network communication or in the actuators. Based on the conventional predictor-based feedback approach, a non-ideal predictor-based control scheme is constructed for each subsystem by using the estimate of the disturbance and the information of the relative state. By exploring certain features of the Laplacian matrix, global consensus analysis is put in the frame- 
work of Lyapunov analysis. The proposed analysis ensures that the integral terms of the system state are carefully considered by using Krasovskii functionals. Sufficient conditions are derived for the Lipschitz nonlinear systems with input delay to guarantee consensus with disturbance rejection in the time domain. Finally, an example is employed to demonstrate the validity of the theoretical results.

\section{Appendix}

Proof of Lemma 6. From the definition of $\Delta_{1}$ in 19 , we have $\left\|\Delta_{1}\right\|^{2}=\sum_{i=2}^{N}\left\|\delta_{i}\right\|^{2}$. With 20$]$, we can get

$$
\delta_{i}=c \gamma_{1} B B^{T} P \sum_{j=2}^{N} l_{i j} \int_{t-h}^{t} e^{A(t-\tau)} \psi_{j} \mathrm{~d} \tau,
$$

and

$$
\begin{gathered}
\left\|\delta_{i}\right\|^{2}=c^{2} \gamma_{1}^{2} \int_{t-h}^{t}\left(\sum_{j=2}^{N} l_{i j} \psi_{j}^{T}\right) e^{A^{T}(t-\tau)} \mathrm{d} \tau P B B^{T} \\
B B^{T} P \int_{t-h}^{t} e^{A(t-\tau)}\left(\sum_{j=2}^{N} l_{i j} \psi_{j}\right) \mathrm{d} \tau .
\end{gathered}
$$

Based on Lemma 3 and the condition [24], one obtains

$$
\left\|\delta_{i}\right\|^{2} \leq c^{2} \gamma_{1}^{2} h \rho^{2} \int_{t-h}^{t} \sum_{j=2}^{N} l_{i j} \psi_{j}^{T} e^{\left(A^{T}+A\right)(t-\tau)} \sum_{j=2}^{N} l_{i j} \psi_{j} \mathrm{~d} \tau .
$$

In light of Lemma 4 and the condition 277 , one gets that

$$
\begin{aligned}
\left\|\delta_{i}\right\|^{2} & \leq(N-1) c^{2} \gamma_{1}^{2} h \rho^{2} e^{\alpha_{2} h} \sum_{j=2}^{N} l_{i j}^{2} \int_{t-h}^{t}\left\|\phi\left(x_{j}\right)-\phi\left(x_{1}\right)\right\|^{2} \mathrm{~d} \tau \\
& \leq(N-1) c^{2} \gamma_{1}^{2} h \rho^{2} e^{\alpha_{2} h} \gamma^{2} \sum_{j=2}^{N} l_{i j}^{2} \int_{t-h}^{t} \xi_{j}^{T}(\tau) \xi_{j}(\tau) \mathrm{d} \tau \\
& \leq(N-1) c^{2} \gamma_{1}^{2} h \rho^{2} e^{\alpha_{2} h} \gamma^{2}\left\|l_{i}\right\|^{2} \int_{t-h}^{t} \xi^{T}(\tau) \xi(\tau) \mathrm{d} \tau .
\end{aligned}
$$

Consequently,

$$
\begin{aligned}
\left\|\Delta_{1}\right\|^{2} & \leq(N-1) c^{2} \gamma_{1}^{2} h \rho^{2} e^{\alpha_{2} h} \gamma^{2} \sum_{i=2}^{N}\left\|l_{i}\right\|^{2} \int_{t-h}^{t} \xi^{T}(\tau) \xi(\tau) \mathrm{d} \tau \\
& \leq(N-1) c^{2} \gamma_{1}^{2} h \rho^{2} e^{\alpha_{2} h} \gamma^{2}\left\|\mathcal{L}_{1}\right\|_{F}^{2} \int_{t-h}^{t} \xi^{T}(\tau) \xi(\tau) \mathrm{d} \tau
\end{aligned}
$$


In a similar way, we have

$$
\bar{\delta}_{i}=c \gamma_{1} B B^{T} P \sum_{j=2}^{N} l_{i j} \int_{t-h}^{t} e^{A(t-\tau)} B F e^{S h} z_{j}(\tau-h) \mathrm{d} \tau .
$$

It follows that

$$
\begin{aligned}
\left\|\bar{\delta}_{i}\right\|^{2} \leq & c^{2} \gamma_{1}^{2} \rho^{2} h \int_{t-h}^{t}\left(\sum_{j=2}^{N} l_{i j} z_{j}^{T}(\tau-h)\right) e^{S^{T} h} F^{T} B^{T} e^{A^{T}(t-\tau)} \\
& \times e^{A(t-\tau)} B F e^{S h}\left(\sum_{j=2}^{N} l_{i j} z_{j}(\tau-h)\right) \mathrm{d} \tau \\
\leq & h \alpha_{1} c^{2} \gamma_{1}^{2} \rho^{2} e^{\left(\alpha_{0}+\alpha_{2}\right) h} \int_{t-h}^{t} \sum_{j=2}^{N} l_{i j} z_{j}^{T}(\tau-h) \sum_{j=2}^{N} l_{i j} z_{j}(\tau-h) \mathrm{d} \tau \\
\leq & (N-1) h \alpha_{1} c^{2} \gamma_{1}^{2} \rho^{2} e^{\left(\alpha_{0}+\alpha_{2}\right) h} \sum_{j=2}^{N} l_{i j}^{2} \int_{t-h}^{t} z_{j}^{T}(\tau-h) z_{j}(\tau-h) \mathrm{d} \tau .
\end{aligned}
$$

Consequently,

$$
\begin{aligned}
\left\|\Delta_{2}\right\|^{2} & \leq(N-1) h \alpha_{1} c^{2} \gamma_{1}^{2} \rho^{2} e^{\left(\alpha_{0}+\alpha_{2}\right) h} \sum_{i=2}^{N}\left\|l_{i}\right\|^{2} \int_{t-h}^{t} z^{T}(\tau-h) z(\tau-h) \mathrm{d} \tau \\
& \leq(N-1) h \alpha_{1} c^{2} \gamma_{1}^{2} \rho^{2} e^{\left(\alpha_{0}+\alpha_{2}\right) h}\left\|\mathcal{L}_{1}\right\|_{F}^{2} \int_{t-h}^{t} z^{T}(\tau-h) z(\tau-h) \mathrm{d} \tau .
\end{aligned}
$$

This completes the proof.

\section{References}

[1] R. Olfati-Saber, R. M. Murray, Consensus problems in networks of agents with switching topology and time-delays, IEEE Trans. Autom. Control 49 (9) (2004) $1520-1533$.

[2] J. A. Fax, R. M. Murray, Information flow and cooperative control of vehicle formations, IEEE Trans. Autom. Control 49 (9) (2004) 1465-1476.

[3] W. Ren, R. W. Beard, Consensus seeking in multi-agent systems under dynamically changing interaction topologies, IEEE Trans. Autom. Control 50 (5) (2005) $655-661$. 
[4] Y. Hong, J. Hu, L. Gao, Tracking control for multi-agent consensus with an active leader and variable topology, Automatica 42 (7) (2006) 1177-1182.

[5] Z. Li, Z. Duan, G. Chen, L. Huang, Consensus of multi-agent systems and synchronization of complex networks: a unified viewpoint, IEEE Trans. Circuits Syst. I, Reg. Papers 57 (1) (2010) 213-224.

[6] Y. Hu, J. Lam, J. Liang, Consensus of multi-agent systems with Luenberger observers, J. Frankl. Inst. 350 (9) (2013) 2769-2790.

[7] Z. Li, H. Ishiguro, Consensus of linear multi-agent systems based on full-order observer, J. Frankl. Inst. 351 (2) (2014) 1151-1160.

[8] Z. Zhao, Z. Lin, Global leader-following consensus of a group of general linear systems using bounded controls, Automatica 68 (2016) 294-304.

[9] J. Sun, Z. Geng, Y. Lv, Adaptive output feedback consensus tracking for heterogeneous multi-agent systems with unknown dynamics under directed graphs, Syst.

1. Control Lett. 87 (2016) 16 -22. doi:http://dx.doi.org/10.1016/j. sysconle.2015.10.007

[10] W. Yu, G. Chen, M. Cao, Consensus in directed networks of agents with nonlinear dynamics, IEEE Trans. Autom. Control 56 (6) (2011) 1436-1441.

[11] Z. Li, W. Ren, X. Liu, M. Fu, Consensus of multi-agent systems with general linear and Lipschitz nonlinear dynamics using distributed adaptive protocols, IEEE Trans. Autom. Control 58 (7) (2013) 1786-1791.

[12] Z. Ding, Consensus control of a class of Lipschitz nonlinear systems, Int. J. Control 87 (11) (2014) 2372-2382.

[13] X. Dong, Q. Li, Z. Ren, Y. Zhong, Formation-containment control for high-order linear time-invariant multi-agent systems with time delays, Journal of the Franklin Institute 352 (9) (2015) 3564 - 3584, special Issue on Synchronizability, Controllability and Observability of Networked Multi-Agent Systems. doi:http://dx.doi.org/10.1016/j.jfranklin.2015.05.008 
URL http://www.sciencedirect.com/science/article/pii/ S0016003215001830

[14] X. Dong, Z. Shi, G. Lu, Y. Zhong, Formation-containment analysis and design for high-order linear time-invariant swarm systems, International Journal of Robust and Nonlinear Control 25 (17) (2015) 3439-3456. doi:10.1002/rnc.3274. URL http://dx.doi.org/10.1002/rnc.3274

[15] J. Lü, F. Chen, G. Chen, Nonsmooth leader-following formation con1 trol of nonidentical multi-agent systems with directed communica255 a tion topologies Automatica 64 (2016) 112 - 120 . doi:http: //dx.doi.org/10.1016/j.automatica.2015.11.004 URL http://www.sciencedirect.com/science/article/pii/ S0005109815004689

[16] X. Dong, Y. Zhou, Z. Ren, Y. Zhong, Time-varying formation control for unmanned aerial vehicles with switching interaction topolo-

a gies, Control Engineering Practice 46 (2016) 26 - 36. doi:http: //dx.doi.org/10.1016/j.conengprac.2015.10.001

aURL http://www.sciencedirect.com/science/article/pii/ S0967066115300289

[17] C. Huang, D. W. C. Ho, J. Lu, J. Kurths, Pinning synchronization in T-S fuzzy complex networks with partial and discrete-time couplings, IEEE Transactions on Fuzzy Systems 23 (4) (2015) 1274-1285. doi:10.1109/TFUZZ.2014. 2350534

[18] B. Wang, J. Wang, L. Zhang, B. Zhang, X. Li, Cooperative control of heterogeneous uncertain dynamical networks: an adaptive explicit synchronization

口 framework, IEEE Transactions on Cybernetics PP (99) (2016) 1-12. doi: $10.1109 /$ TCYB.2016.2549556.

[19] L. Li, D. W. C. Ho, J. Cao, J. Lu, Pinning cluster synchronization in an array of coupled neural networks under event-based mechanism, Neural Networks 76 
(2016) 1 - 12. doi:http://dx.doi.org/10.1016/j.neunet.2015. 12.008 .

URL http://www.sciencedirect.com/science/article/pii/ S0893608015002774

[20] J. Lu, C. Ding, J. Lou, J. Cao, Outer synchronization of partially coupled dynamical networks via pinning impulsive controllers Journal of

a the Franklin Institute 352 (11) (2015) 5024 - 5041. doi:http: $/ / \mathrm{dx}$. doi.org/10.1016/j.jfranklin.2015.08.016

a URL http://www.sciencedirect.com/science/article/pii/ S0016003215003373

[21] B. Wang, J. Wang, B. Zhang, X. Li, Global cooperative control framework for multiagent systems subject to actuator saturation with industrial applications, IEEE Transactions on Systems, Man, and Cybernetics: Systems PP (99) (2016) 1-14. doi:10.1109/TSMC.2016.2573584

[22] G. Wen, Z. Duan, G. Chen, W. Yu, Consensus tracking of multi-agent systems with Lipschitz-type node dynamics and switching topologies, IEEE Transactions on Circuits and Systems I: Regular Papers 61 (2) (2014) 499-511. doi:10. $1109 /$ TCSI.2013.2268091.

[23] Y. Liu, Y. Jia, $\mathrm{H}_{\infty}$ consensus control for multi-agent systems with linear coupling dynamics and communication delays, Int. J. Syst. Sci. 43 (1) (2012) 50-62.

[24] G. Wen, W. Yu, M. Z. Chen, X. Yu, G. Chen, $\mathrm{H}_{\infty}$ pinning synchronization of directed networks with aperiodic sampled-data communications, IEEE Trans. Circuits Syst. I, Reg. Papers 61 (11) (2014) 3245-3255.

[25] L. Guo, W.-H. Chen, Disturbance attenuation and rejection for systems with nonlinearity via DOBC approach, Int. J. Robust Nonlinear Control 15 (3) (2005) $109-125$.

[26] S. Li, J. Yang, W.-H. Chen, X. Chen, Disturbance observer-based control: methods and applications, CRC press, 2014. 
[27] H. Du, S. Li, P. Shi, Robust consensus algorithm for second-order multi-agent systems with external disturbances, Int. J. Control 85 (12) (2012) 1913-1928.

[36] C. Wang, Z. Zuo, Z. Lin, Z. Ding, Consensus control of a class of Lipschitz nonlinear systems with input delay, IEEE Trans. Circuits Syst. I, Reg. Papers 62 (11) (2015) 2730-2738. doi:10.1109/TCSI.2015.2479046 
[37] Y. Chen, D. W. C. Ho, J. Lü, Z. Lin, Convergence rate for discrete-time multiagent systems with time-varying delays and general coupling coefficients, IEEE Trans.

1 Neural Netw. Learn. Syst. 27 (1) (2016) 178-189. doi:10.1109/TNNLS. 2015.2473690

[38] M. Krstic, Delay compensation for nonlinear, adaptive, and PDE systems, Springer, 2009.

[39] Z. Li, Z. Duan, Cooperative Control of Multi-Agent Systems: A Consensus Region Approach, CRC Press, 2014.

[40] Z. Li, G. Wen, Z. Duan, W. Ren, Designing fully distributed consensus protocols for linear multi-agent systems with directed graphs, IEEE Trans. Autom. Control 60 (4) (2015) 1152-1157.

[41] W. Kwon, A. Pearson, Feedback stabilization of linear systems with delayed control, IEEE Trans. Autom. Control 25 (2) (1980) 266-269.

[42] Z. Artstein, Linear systems with delayed controls: a reduction, IEEE Trans. Autom. Control 27 (4) (1982) 869-879.

[43] K. Gu, J. Chen, V. L. Kharitonov, Stability of time-delay systems, Springer Science \& Business Media, 2003.

[44] S. Y. Yoon, P. Anantachaisilp, Z. Lin, An LMI approach to the control of exponentially unstable systems with input time delay, in: 52nd IEEE Conference

1. on Decision and Control, 2013, pp. 312-317. doi:10.1109/CDC.2013. 6759900

[45] Z. Ding, Z. Lin, Truncated state prediction for control of Lipschitz nonlinear systems with input delay, in: 53rd IEEE Conference on Decision and Control, 2014, pp. 1966-1971. doi:10.1109/CDC.2014.7039686.

[46] C. Wang, Z. Zuo, Z. Lin, Z. Ding, A truncated prediction approach to consensus control of Lipschitz nonlinear multi-agent systems with input delay, IEEE Transactions on Control of Network Systems PP (99) (2016) 1-1. doi:10.1109/ TCNS.2016.2545860, 